\title{
PATTERNS OF PROSTATIC INTRAEPITHELIAL NEOPLASIA
}

\author{
Dukkipati Kalyani1, Ramanavarapu Sasank ${ }^{2}$
}

${ }^{1}$ Associate Professor, Department of Pathology, Siddhartha Medical College, Vijayawada, Andhra Pradesh.

${ }^{2}$ Professor, Department of Pathology, Siddhartha Medical College, Vijayawada, Andhra Pradesh.

ABSTRACT
BACKGROUND
Prostatic intraepithelial neoplasia (PIN) remains as a well-known precancerous condition. Identification in biopsy specimen
warrants repeat biopsy for subsequent carcinoma. The main aim of the study is to describe the morphological spectrum of
prostatic intraepithelial neoplasia (PIN), its relationship to carcinoma of the prostate and its clinical significance.

\section{MATERIALS AND METHODS}

This is a descriptive study. Patients who were diagnosed clinically as benign prostatic hyperplasia (BPH) with symptoms of obstruction (hesitancy, poor flow, intermittent stream, dribbling) were included. Sections of the TURP chips were reviewed retrospectively in the Department of Pathology, Siddhartha Medical College, Vijayawada. A total of 75 cases were studied during a period of 2 years (October 2015 to November 2017) from the age group between 40 - 90 years.

\section{RESULTS}

75 cases of TURP specimens were studied. Majority of cases are PIN, 43 cases (58\%). All PIN cases were graded into 22 cases of low-grade PIN (LGPIN) (51\%) and 21 cases of high-grade PIN (HGPIN) (49\%). HGPIN shows marked nuclear enlargement, prominent eosinophilic nucleoli and increased chromatin compared to low-grade PIN. Majority of HGPIN cases in our study were noted in 60 - 69 yrs. constituting about 30 cases.

\section{CONCLUSION}

As prostatic intraepithelial neoplasia is a precursor lesion for prostatic carcinoma, it needs to be detected as early as possible. Prostatic biopsy helps to identify and treat the patients with high-grade PIN as well as carcinoma.

\section{KEYWORDS}

Prostate Cancer, Prostatic Intraepithelial Neoplasia, TURP.

HOW TO CITE THIS ARTICLE: Kalyani D, Sasank R. Patterns of prostatic intraepithelial neoplasia. J. Evolution Med. Dent. Sci. 2018;7(04):399-402, DOI: 10.14260/jemds/2018/89

\section{BACKGROUND}

Prostatic intraepithelial neoplasia (PIN) remains as a wellknown precancerous condition. Identification in biopsy specimen warrants repeat biopsy for subsequent carcinoma. PIN does not elevate serum PSA concentration, palpable mass or detected on ultrasound. Thus, the only method of identification is biopsy. The significance of high-grade PIN is mainly to identify the patients at risk for prostatic carcinoma. Increased use of biopsy protocols are more likely to identify high-grade PIN and less likely to miss concurrent carcinoma.[1]

\section{Aims and Objectives}

The main aim of the study is to describe the morphological spectrum of PIN, its relationship to carcinoma of the prostate and its clinical significance.

\section{MATERIALS AND METHODS}

This is a descriptive study. Patients who were diagnosed clinically as BPH with symptoms of obstruction (hesitancy,

'Financial or Other Competing Interest': None.

Submission 08-12-2017, Peer Review 05-01-2018,

Acceptance 11-01-2018, Published 20-01-2018.

Corresponding Author:

Dr. Ramanavarapu Sasank,

Professor, Department of Pathology,

Siddhartha Medical College,

Vijayawada-521108, Andhra Pradesh.

E-mail: drsasank@gmail.com

DOI: $10.14260 /$ jemds $/ 2018 / 89$

(c) (i) $\odot$ poor flow, intermittent stream, dribbling) were included in this study. Sections of the TURP chips were reviewed retrospectively in the Department of Pathology, Siddhartha Medical College, Vijayawada. A total of 75 cases were studied during a period of 2 years (October 2015 to November 2017) from the age groups between 40 - 90 years.

\section{RESULTS}

75 cases of TURP specimens were studied. Various lesions identified were BPH, nonspecific prostatitis, PIN and prostatic carcinoma as shown in Table 1 . Among these, 43 cases (58\%) of PIN were diagnosed based on the presence of ductal/ acinar epithelial changes including nuclear enlargement, prominent nucleoli, chromatin alteration and luminal complexity. HGPIN and Prostatic carcinoma shared increased incidence and severity with advancing age. Majority of HGPIN cases in our study were noted in (60 - 80 years) as shown in Table 2. Out of 43 cases of PIN LGPIN constitutes about 22 cases (51\%), whereas HGPIN were 21 cases (49\%) as shown in Table 3. HGPIN shows marked nuclear enlargement, prominent eosinophilic nucleoli and increased chromatin compared to low-grade PIN (Fig. 1, 2). The nuclei of the cells that make up LGPIN are enlarged, vary in size, have a normal or slightly increased chromatin content and possess small or inconspicuous nucleoli (Fig. 3, 4). HGPIN is characterised by cells with large nuclei of relatively uniform size, an increased chromatin content which may be irregularly distributed and prominent nucleoli that are similar to those of carcinoma cells (Fig. 5, 6). Mitotic figures are rare in HGPIN and are not included in the grading criteria for PIN. 


\begin{tabular}{|c|c|c|}
\hline Lesion & No. of Cases & Percentage \\
\hline PIN & 43 & $58 \%$ \\
\hline Carcinoma & 14 & $18 \%$ \\
\hline BPH & 14 & $18 \%$ \\
\hline Non-Specific & 4 & $6 \%$ \\
\hline Total & $\mathbf{7 5}$ & $\mathbf{1 0 0 \%}$ \\
\hline \multicolumn{2}{|r|}{ Table 1. Various Lesions of Prostate }
\end{tabular}

\begin{tabular}{|c|c|c|c|c|c|}
\hline $\begin{array}{c}\text { Age Group } \\
\text { (yrs.) }\end{array}$ & $\begin{array}{c}\text { Non- } \\
\text { Specific }\end{array}$ & BPH & Carcinoma & PIN & Total \\
\hline $40-49$ & 1 & - & 2 & 1 & 4 \\
\hline $50-59$ & - & 2 & - & 4 & 6 \\
\hline $60-69$ & 3 & 9 & 9 & 30 & 51 \\
\hline $70-79$ & - & 1 & 3 & 8 & 12 \\
\hline $80-89$ & - & 2 & - & - & 2 \\
\hline Total & $\mathbf{4}$ & $\mathbf{1 4}$ & $\mathbf{1 4}$ & $\mathbf{4 3}$ & $\mathbf{7 5}$ \\
\hline Table 2. Distribution of Prostatic Lesions by Age Groups \\
\hline
\end{tabular}

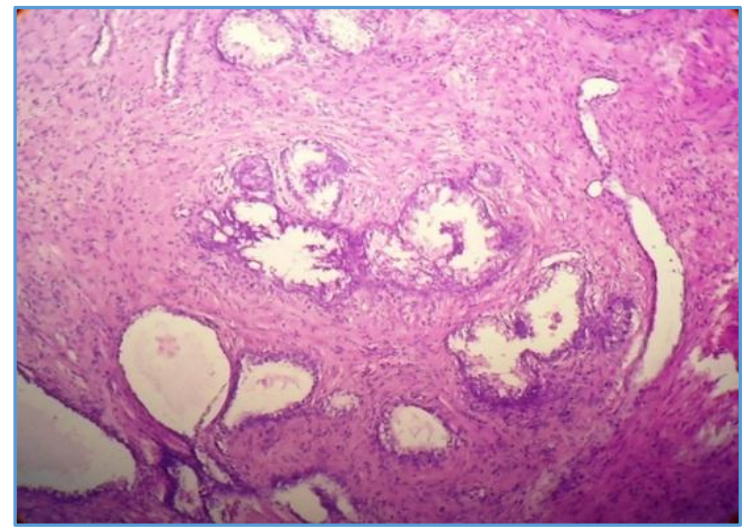

Figure 3. Low-Grade PIN 10X

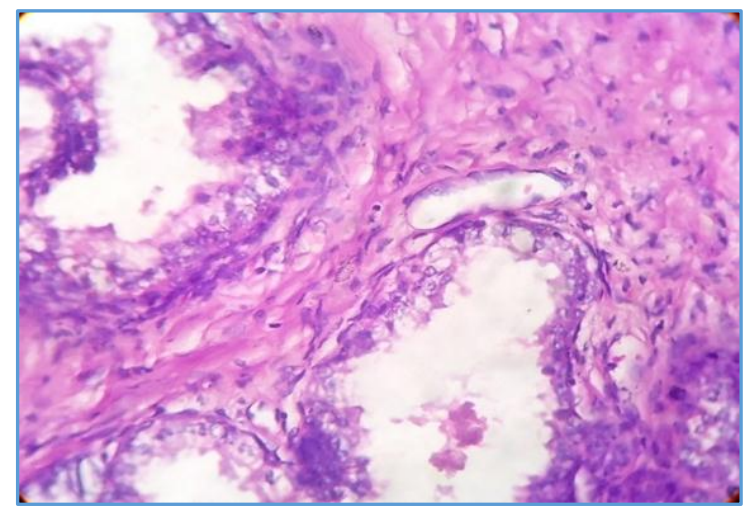

Figure 4. Low-Grade PIN 40X

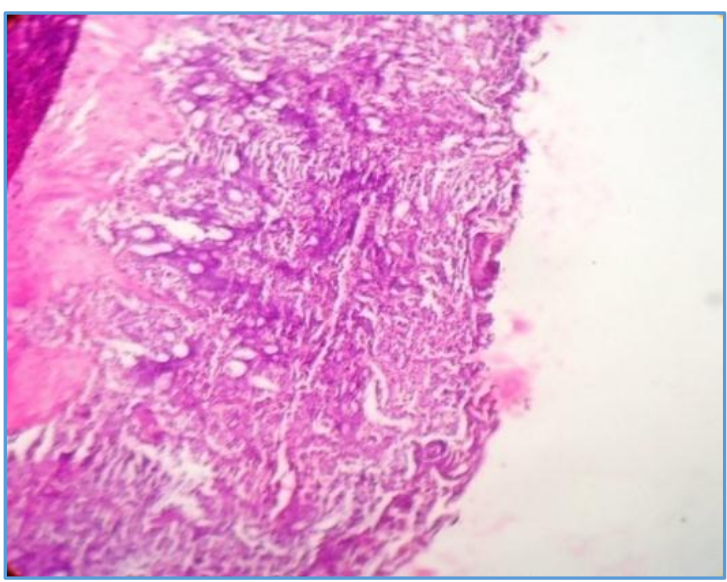

Figure 5. Adenocarcinoma 10X

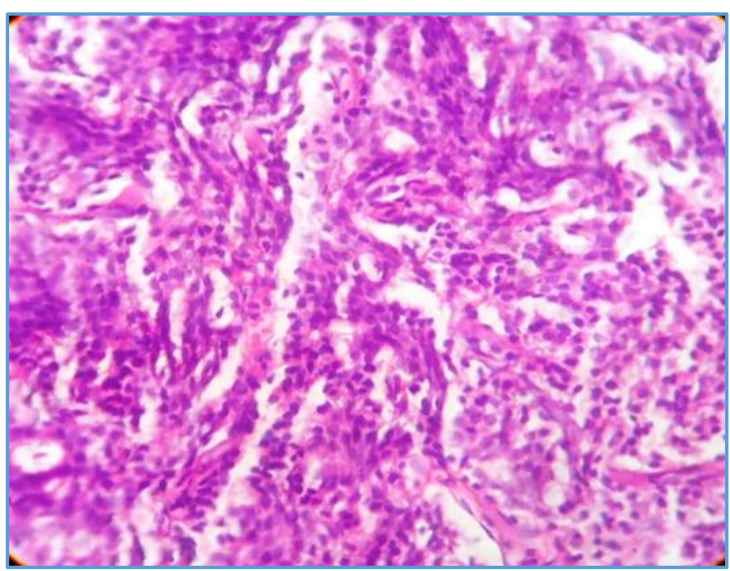

Figure 6. Adenocarcinoma $40 \mathrm{X}$

Figure 2. High-Grade PIN 40X 


\section{DISCUSSION}

We reviewed the cases of premalignant lesions of the prostate with an emphasis on high-grade prostatic intraepithelial neoplasia (HGPIN). Out of 75 cases, majority of the cases were prostatic intraepithelial 43 cases (58\%). Out of these, LGPIN were 23 cases (51\%) and HGPIN were 21 cases (49\%). The classification of PIN into low grade and high grade is chiefly based on the cytological characteristics of the secretory cells. HGPIN is characterised by cellular proliferations within pre-existing ducts and acini with nuclear and nucleolar enlargement similar to prostatic carcinoma. The clinical importance of recognising HGPIN is based on its association with prostatic carcinoma. In recent years, a significant decline from $36 \%$ to $22 \%$ in the predictive value of cancer after an initial diagnosis of HGPIN. A major factor contributing to the decreased incidence of cancer after a diagnosis of HGPIN on needle biopsy in the contemporary era is related to increased needle biopsy core sampling, which detects many associated cancers on initial biopsy. Multifocal HGPIN when isolated in a prostate biopsy still carries a high predictive value for carcinoma in repeat biopsy.[2-5]

Although, the cytological features of LGPIN and HGPIN are fairly constant, the architecture shows a spectrum varying from a flattened epithelium to a florid cribriform proliferation. There are four main patterns of HGPIN, a tufting pattern, a micropapillary pattern, a cribriform pattern and a flat pattern. Variants of HGPIN include Signet ring-cell, small cell neuroendocrine, mucinous, foamy, inverted and a variant with squamous differentiation. The presence of HGPIN with various histological patterns further supports the hypothesis that there is a close relationship between HGPIN and the variants of prostatic carcinoma.[6-8] The central zone glands are architecturally more complex than the peripheral and transition zone glands and exhibit a certain degree of nuclear stratification that may be interpreted as PIN. In addition bridging, papillary formation with a central vascular core and focal tubular or cribriform patterns may be present.

HGPIN has to be differentiated from prostatic carcinoma, clear cell cribriform hyperplasia (CCCH) and basal cell hyperplasia. They are typically located in the transition zone and typically although not always sampled on TURP, whereas HGPIN predominates in the peripheral zone. Finally, most cases of BCH are found in TURP specimens indicating growth in the transition zone in contrast to HGPIN's preferential location in the periphery.

HGPIN is the precursor lesion to some forms of adenocarcinoma. HGPIN should not be confused with intraductal carcinoma. The latter, even when isolated in a prostate biopsy, carries a predictive value of $100 \%$ for cancer in a repeat biopsy. Intraductal carcinoma is characterised by a proliferation of malignant epithelial cells filling large acini and ducts with complete or incomplete retention of basal cells. It may show solid, dense cribriform, loose cribriform and micropapillary patterns and generally occurs in conjunction with invasive cribriform or ductal adenocarcinoma of the prostate. While the most common forms of invasive ductal adenocarcinoma mimic micropapillary and cribriform HGPIN, ductal adenocarcinoma may be composed of simple glands lined by stratified columnar epithelium with cytological and architectural features of flat and tufting HGPIN, i.e. PIN-like ductal cancers. PIN-like ductal cancers are distinguished from HGPIN, either because the atypical glands are too crowded to represent HGPIN or there are too many atypical glands.

The cells of Transitional cell carcinoma (TCC) usually vary significantly in size and shape and have a very coarse chromatin pattern, significant mitotic activity and frequent tumour necrosis. In lower grade TCC, the cells may show longitudinal nuclear grooves. Because TCC in situ of the bladder frequently shows pagetoid spread, prostatic ducts involved by TCC frequently have a basal cell layer as in HGPIN. Mitotic figures are frequently present in high-grade TCC and in prostate carcinoma of ductal origin, and are rare in cribriform carcinoma and in HGPIN.

Low-grade PIN on biopsy should not be listed in pathology reports, because there is a lack of reproducibility in its diagnosis, even by uropathologists, and it is not associated with a higher risk of cancer on re-biopsy than the risk after a benign diagnosis on initial biopsy.

Risk of cancer on re-biopsy studies from the early $1990 \mathrm{~s}$ on relatively few cases reported a $50 \%$ risk of cancer after the diagnosis of HGPIN. On re-biopsy some of these initially missed cancers were detected, yielding a high re-biopsy risk of cancer. More contemporary data report that the median risk of cancer after a diagnosis of HGPIN on biopsy is only $22 \%$. This is similar to the median risk of finding cancer in a repeat biopsy after a benign diagnosis, which is 15\% - 19\%.

In cases with HGPIN and adjacent small atypical glands, where the differential diagnosis of the small glands is adjacent cancer or outpouchings of the HGPIN, the risk of cancer is equivalent to that after a diagnosis of atypical glands suspicious for carcinoma. The morphology of HGPIN (flat vs. tufting vs. micropapillary vs. cribriform) does not determine which HGPIN lesions are at greater risk of being associated with carcinoma on repeat biopsy.

Re-biopsy should be performed in the region of the original HGPIN site and in adjacent sites, although the entire prostate should be sampled. There is epidemiological, morphological and molecular evidence that HGPIN is a precursor lesion to some carcinomas of the prostate. The clinical importance of recognising HGPIN is based on its association with Prostatic carcinoma. The results were compared with similar other studies.(1,8-15) In our study, 58\% of the PIN cases were identified similar to other studies. In recent years, a significant decline from $36 \%$ to $22 \%$ in the predictive value of Prostatic carcinoma after an initial diagnosis of HGPIN has been observed. A major factor contributing to the decreased incidence of Prostatic carcinoma after a diagnosis of HGPIN on needle biopsy in the contemporary era is related to increased needle biopsy core sampling, which detects many associated cancers on initial biopsy.

\section{CONCLUSION}

As prostatic intraepithelial neoplasia is a precursor lesion for prostatic carcinoma, it needs to be detected as early as possible. Prostatic biopsy helps to identify and treat the patients with high-grade PIN as well as carcinoma. Re-biopsy should be obtained in the patients in high-grade PIN to detect adjacent carcinoma. 


\section{REFERENCES}

[1] McNeal JE, Bostwick DG. Intraductal dysplasia: a premalignant lesion of the prostate. Hum Pathol 1986;17(1):64-71.

[2] Ayala AG, Ro JY. Prostatic intraepithelial neoplasia: recent advances. Arch Pathol Lab Med 2007;131(8):1257-66.

[3] Epstein JI. Precursor lesions to prostatic adenocarcinoma. Virchows Arch 2009;454(1):1-16.

[4] Montironi R, Mazzucchelli R, Lopez-Beltran A, et al. Mechanisms of disease: high-grade prostatic intraepithelial neoplasia and other proposed preneoplastic lesions in the prostate. Nat Clin Pract Urol 2007;4(6):321-32.

[5] Montironi R, Mazzucchelli R, Scarpelli M. Precancerous lesions and conditions of the prostate: from morphological and biological characterization to chemoprevention. Ann N Y Acad Sci 2002;963:169-84.

[6] Kumaresan K, Kakkar N, Verma A, et al. Diagnostic utility of alpha-methylacyl CoA racemase (P504S) \& HMWCK in morphologically difficult prostate cancer. Diagn Pathol 2010;5:83.

[7] Wang X, Hickey RJ, Malkas LH, et al. Elevated expression of cancer-associated proliferating cell nuclear antigen in high-grade prostatic intraepithelial neoplasia and prostate cancer. Prostate 2011;71(7):748-54.

[8] Bostwick DG, Ma J. Over-diagnosis of high-grade prostatic intraepithelial neoplasia: a prospective study of 251 cases. BJU Int 2007;100(5):1036-9.
[9] Cohen RJ, Shannon BA, Weinstein SL. Intraductal carcinoma of the prostate gland with transmucosal spread to the seminal vesicle: a lesion distinct from high-grade prostatic intraepithelial neoplasia. Arch Pathol Lab Med 2007;131(7):1122-5.

[10] Tavora F, Epstein JI. High-grade prostatic intraepithelial neoplasia like ductal adenocarcinoma of the prostate: a clinicopathologic study of 28 cases. Am J Surg Pathol 2008;32(7):1060-7.

[11] Mosquera JM, Perner S, Genega EM, et al. Characterization of TMPRSS2-ERG fusion high-grade prostatic intraepithelial neoplasia and potential clinical implications. Clin Cancer Res 2008;14(11):3380-5.

[12] Eskicorapci SY, Guliyev F, Islamoglu E, et al. The effect of prior biopsy scheme on prostate cancer detection for repeat biopsy population: results of the 14-core prostate biopsy technique. Int Urol Nephrol 2007;39(1):189-95.

[13] Srigley JR, Merrimen JL, Jones G, et al. Multifocal highgrade prostatic intraepithelial neoplasia is still a significant risk factor for adenocarcinoma. Can Urol Assoc J 2010;4(6):434.

[14] Epstein JI, Herawi M. Prostate needle biopsies containing prostatic intraepithelial neoplasia or atypical foci suspicious for carcinoma: implications for patient care. J Urol 2006;175(3 Pt 1):820-34.

[15] Laurila $M$, van der Kwast $T$, Bubendorf $L$, et al. Detection rates of cancer, high grade PIN and atypical lesions suspicious for cancer in the European Randomized Study of Screening for Prostate Cancer. Eur J Cancer 2010;46(17):3068-72. 